\title{
Description of condyle trabecular bone density of hypertension patients evaluated from panoramic radiograph using software imageJ
}

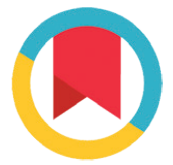

CrossMark

\author{
Meyta R. Gwen, ${ }^{*}$ Ria N. Firman, Farina Pramanik
}

\section{Abstract}

Objective: The purpose of this research is to find condyle trabecular bone density of hypertension patients evaluating from panoramic radiograph using software imageJ.

Material and Methods: The type of research is descriptive using purposive sampling method, 25 panoramic radiographs of hypertension patients aged 25-45 years old are obtained. The condyle trabecular analysis is done using software ImageJ with region of interest (ROI) $50 \times 50$ pixel.
Results: This shows that the condyle trabecular bone density of hypertension patients evaluated from panoramic radiograph using software ImageJ in trabecular area $29.929 \%$ and marrow area $70.071 \%$.

Conclusion: This research is that the condyle trabecular on hypertension patients evaluated from panoramic radiograph using software ImageJ was decreasing.
Department of Dental Radiology, Faculty of Dentistry, Padjajaran University, Bandung, Indonesia
*Correspondence to: Meyta Radhila Gwen, Department of Dental Radiology, Faculty of Dentistry, Padjajaran University, Bandung, Indonesia meytaradhilagwen@gmail.com

Received: 22 February 2017 Revised: 24 May 2017 Accepted: 9 August 2019 Available Online: 1 August 2020

Keywords: Bone density, Condyle, Hypertension, Panoramic radiograph trabecular

Cite this Article: Gwen MR, Firman RN, Pramanik F. 2020. Description of condyle trabecular bone density of hypertension patients evaluated from panoramic radiograph using software imageJ. Journal of Dentomaxillofacial Science 5(2): 77-81. D0I: 10.15562/jdmfs.v5i2.441

\section{Introduction}

Trabecular density seen by the presence and thickness of hard tissue in the form of nets bone that restrict the marrow cavity or so-called trabecular nets. ${ }^{1}$ Mandibular condyle is an important part to be a region of interest (ROI), because it contains a lot of trabecular (98.6\%). ${ }^{2}$ Previous research states that by trabecular can be used to detect changes in the quality of their bones by $94 \%$ compared with cortical is $64 \% .^{1}$ Research on trabecular pattern has been done on patients with a history of systemic disorders, particularly by using panoramic radiograph. ${ }^{3}$ Hypertension is a systemic disease in which there is an increase in blood pressure continuously to exceed the normal limits, normal blood pressure is 140/90 mmHg. ${ }^{4}$ Hypertension has become a major public health problem in Indonesia as well as in several countries in the world. In the result of Riskesdas 2013 the prevalence of hypertension $\geq 18$ years of age reached $25.8 \%$ of the total population in Indonesia. ${ }^{5}$

Research conducted a connection between adequate intakes of calcium with a degree of hypertension. The result of the relationship to the disorder of bone density caused by the intake of calcium and vitamin $\mathrm{D}$. The impact on existing systems in the body due to lack of calcium and vitamin $\mathrm{D}$ in the body causes a decrease in bone density systemically. ${ }^{6-8}$ Evidence from clinical and epidemiological studies support a possible association between low vitamin D levels with hypertension. Vitamin D is involved in bone formation, resorption and mineralization as well as in maintaining neuromuscular function primarily responsible for regulating the efficiency of calcium absorption in the intestine. Decreased bone density can occur because of the imbalance of calcium caused one of which is the reduction in calcium absorption by the intestine that caused by changes in the metabolism of vitamin $\mathrm{D}$ and causing disruption of metabolic bone processes. ${ }^{9}$

The results of the study state that panoramic radiographs can assess bone density in macrostructure and microstructure. This is used by researchers to determine the level of decrease in trabecular bone density, one of which is in the condyle. ${ }^{3}$ The use of ImageJ software in digital image analysis has been used extensively in the fields of health and biology, because ImageJ software can be downloaded freely and is capable of displaying, editing, analyzing, processing, storing, and printing images both in DICOM and JPEG formats. ${ }^{10}$

The purpose of this study was to determine the condylus trabecular bone density of hypertensive patients in terms of panoramic radiographs using Image J software.

\section{Material and Methods}

This type of research is descriptive conducted in October-December 2016. After receiving permission 
from the Health Research Ethics Committee of Faculty of Medicine, Padjadjaran University, then carry out research at Denstistry Hospital of Padjadjaran University, Bandung.

The population of this research is all secondary data panoramic radiographs digital of patients with hypertension in radiology installation of Dentistry Hospital of Padjadjaran University, Bandung. Samples were selected by purposive sampling method with criteria: radiographs of hypertensive patients aged 20-50 years, a panoramic radiograph with good quality, condyle clearly visible and can be assessed, there is no picture superimposed on the condyles, there is no fracture in the head condyle. There are 60 populations and 25 samples.

The studies conducted on the surface of the condyle head right and left, by means of: points A and $\mathrm{B}$ are connected by drawing a horizontal line, the center point of condyle heads $(\mathrm{O})$ are in the middle of the line horinzotal $\mathrm{A}$ and $\mathrm{B}$, then drag the vertical line OC perpendicular from the line $\mathrm{AB}$, putting a box measuring $50 \times 50$ pixel box with the center point coincides with the center point and parallel to the line OC figure $1 .^{11}$

Panoramic radiography processed in Software ImageJ accordance with the following steps: a panoramic radiograph already croped and then do the filteralitation. Click process $\rightarrow$ filters $\rightarrow$ gaussian blur and then save with a different file
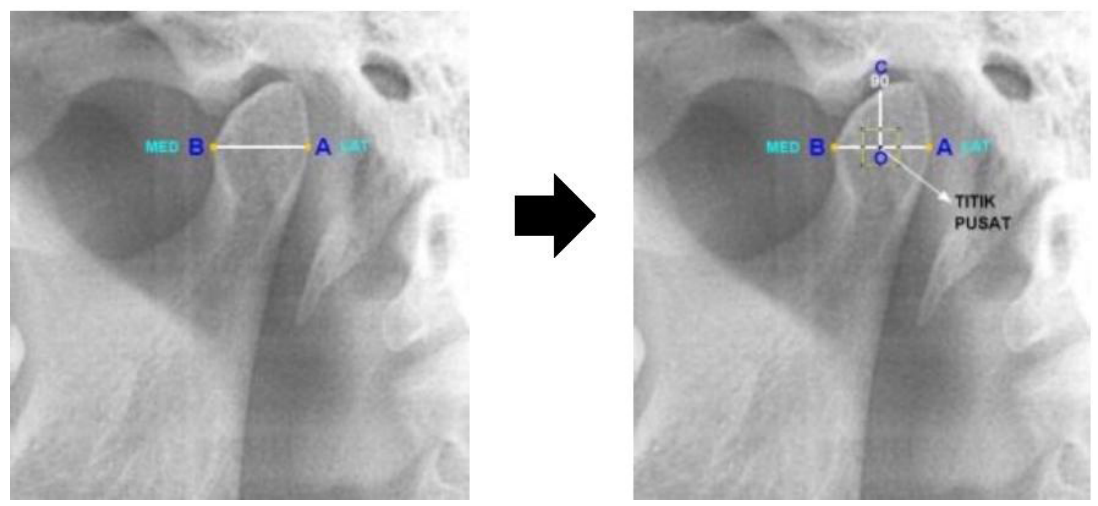

Figure 1 Creating a Horizontal Line AB, Centre Point and Guidelines Box $50 \times 50$ pixels
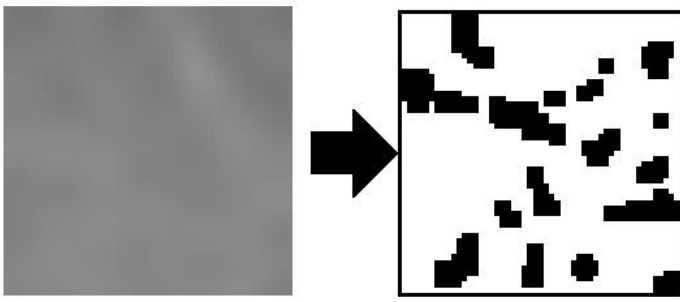

B

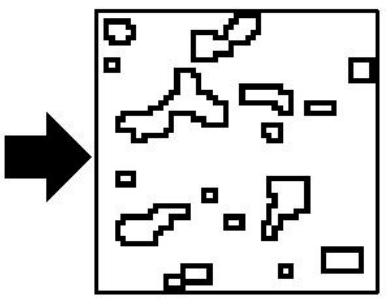

C
Figure 2 Sample Results Filteralitation Process with Gaussian Blur (A), Make Binary Process (B), and Binary Outline Process (C) name, the image that already in the process of filteralitation then do the extract features. Click Process $\rightarrow$ Binary $\rightarrow$ Make Binary, then get the outline of the particle form with click Process $\rightarrow$ Binary $\rightarrow$ Outline, then click analyze and select analyze particle and and then going out results and the percentage of trabecular and marrow in condyle area figure 2.

\section{Results}

The results of the research in the form of a data sample of men and women by age group divisions as follows:

Table 1 shows all samples that collected in the percentage of trabecular bone density of patients with hypertension. There are 25 people with hypertension, with the number of men is 13 and women 12 . The highest number of samples as much as 7 samples of men in the age group 46-55, and the lowest 0 samples in the age group 17-25. The highest number of samples as many as 4 samples of women in the age group 46-55, and the lowest 2 samples in the age group 26-35. In the age group $36-45$, men and women have the same number of samples as much as 3 samples.

Grouping of samples patients with hypertension in Table 1 will be processed and the results will be tabulated in table 2 .

Table 2 shows the results of the average percentage trabecular patients with hypertension right condyle highest in the age group 46-55 (30.203\%) and the lowest in the age group 26-35 (28.797\%). Meanwhile, in the left condyle average percentage of trabecular the highest in the age group 36-45 (31.013\%) and the lowest in the age group 26-35 (28.647\%). The average result percentage marrow patients with hypertension table 2 is the highest in the right condyle in the age group 26-35 (71.203\%) and the lowest in the age group 46-55 (69.797\%). Meanwhile, in the left condyle average percentage of marrow the highest in the age group 26-35 (71.353\%) and the lowest in the age group 36-45 (68.987\%).

Grouping of samples patients with hypertension in table 1 will also be processed to get the results that will be tabulated in table 3 .

Table 3 shows the results of the average percentage of trabecular patients with hypertension, on the right condyle men is higher (30.627\%) compared to women $(28.478 \%)$. Meanwhile, the average percentage of trabecular patients with hypertension on the left condyle, men is lower $(29.482 \%)$ than women $(30.493 \%)$. The average percentage of marrow patients with hypertension on the right condyle, men is lower (69.373\%) than women $(71.518 \%)$. Meanwhile, the average percentage of marrow patients with hypertension 
Table 1 Men and women by age group category

\begin{tabular}{lccc}
\hline & \multicolumn{2}{c}{ Hypertension } & \\
\cline { 2 - 3 } Age & Men & Women & Total \\
\hline $17-25$ & - & 3 & 3 \\
$26-35$ & 3 & 2 & 5 \\
$36-45$ & 3 & 3 & 6 \\
$46-55$ & 7 & 4 & 11 \\
Total & 13 & 12 & 25 \\
\hline
\end{tabular}

Table 2 Average percentage of trabecular and marrow patients with hypertension in condyle right and left by age group

\begin{tabular}{lccccc}
\hline & \multicolumn{4}{c}{ Hypertension } \\
\cline { 2 - 3 } Age & \multicolumn{2}{c}{ (\%) Trabekular } & & \multicolumn{2}{c}{ (\%) Marrow } \\
\cline { 2 - 3 } \cline { 5 - 6 } & Right Condyle & Left Condyle & & Right Condyle & Left Condyle \\
\hline $17-25$ & 28.987 & 30.640 & & 71.013 & 69.360 \\
$26-35$ & 28.797 & 28.647 & & 71.203 & 71.353 \\
$36-45$ & 29.860 & 31.013 & & 70,140 & 68.987 \\
$46-55$ & 30.203 & 29.745 & 69.797 & 70.255 \\
\hline
\end{tabular}

Table 3 Average percentage of trabecular patients with hypertension men and women on the right and left condyle

\begin{tabular}{lccccc}
\hline & \multicolumn{4}{c}{ Hypertension } \\
\cline { 2 - 3 } Age & \multicolumn{2}{c}{ (\%) Trabekular } & & \multicolumn{2}{c}{ (\%) Marrow } \\
\cline { 2 - 3 } \cline { 5 - 6 } Men & 30.627 & 29.482 & & 69.373 & 70.518 \\
Women & 28.478 & 30.493 & & 71.522 & 69.507 \\
\hline
\end{tabular}

Table 4 Average percentage of trabecular and marrow patients with hypertension in right and left condyle

\begin{tabular}{lccc}
\hline & \multicolumn{2}{c}{ Hypertension } & \\
\cline { 2 - 3 } & (\%) Trabekular & (\%) Marrow & (\%) Difference \\
\hline Right Condyle & 29.720 & 70.280 & 40.560 \\
Left Condyle & 30.138 & 69.862 & 39.724 \\
(\%) Difference & \multicolumn{3}{c}{0.418} \\
\hline
\end{tabular}

on the left condyle, men is higher $(70.518 \%)$ than women $(69.507 \%)$.

Grouping of samples patients with hypertension in table 1 will also be processed to get the results that will be tabulated in table 4 .

Table 4 shows the results of the average percentage of trabecular on the right condyle is lower $(29.720 \%)$ compared to left condyle (30.138\%) in patients with hypertension. Meanwhile, the average percentage of marrow on the right condyle is higher $(70.280 \%)$ compared to the right condyle $(69.862 \%)$. Difference in average percentage of trabecular and marrow in right and left condyle patients with hypertension is $0.418 \%$.

\section{Discussion}

Based on it can be seen fairly balanced ratio between the number of samples of men and women overall, but there are differences in sample size of men and women in the age group 17-25 and 46-55 as much as 3 samples and the age group 36-45 there is a difference 1 samples. It shows the number of of samples men and women in that age group is not balanced. It can be caused by several things, including the fact many types and sizes of population and limitations by the researchers.

Based on shows the bone density in early adult age group (26-35) in the right and left condyle is lower than the end adult age group (36-45) and elderly early age group (46-55). According to the research results Saufika, et al (2012) that shows the bone density in early adult age group (26-45) has decreased due to the lifestyle indicated by early adult age groups. Early adult age groups are less likely to eat foods with high calcium and potassium, but like eating foods high in sodium. Calcium intake lack of demand can cause a reduction in bone density.,

Women have a higher risk of osteoporosis than men in the amount of 12.083 times, because the women's bone mass is generally 4 times smaller than men. While women also experience a decrease in bone mass more rapidly than men, especially related to estrogen levels in women. ${ }^{7}$ This is according with the results of the study authors in table 3 shows the results of the average percentage of trabecular patients with hypertension in right condyle of men is higher $(30.627 \%)$ than right condyle of women $(28.478 \%)$ and the average percentage of marrow patients with hypertension in right condyle men is lower $(69.373 \%)$ than women $(71.518 \%)$. While the results of the average percentage of trabecular patients with hypertension in the left condyle indicates a mismatch because the average percentage of trabecular patients with hypertension, men is lower $(29.482 \%)$ than women $(30.493 \%)$ and the average percentage of marrow patients with hypertension, men condyle is higher $(70.518 \%)$ than women (69.507\%).

The difference of average percentage of trabecular and marrow patients with hypertension at the right and left condyle. This shows that there is a tendency to chew on one side, because the rate of bone remodeling is different for each person and it is assumed related to the amount of mechanical loading. The results showed the left condyle has a higher average trabecular, it indicates the left side is more commonly used during mastication. In general, 
the bone that bears heavier weights is estimated to have a higher level of remodeling so the bone more mineralized. The results are consistent the trabecular bone that more dense wil be more mineralized. ${ }^{11}$

Trabecular seen as a radiopaque fine webs on spongy bone which is reflected from radiographic imaging. Widening of the cavity of trabecular patients with hypertension caused by disorders of calcium metabolism that occurs in people with hypertension and lead to higher production of parathyroid hormone $(\mathrm{PTH})$ in patients with hypertension that would trigger an increase in intracellular calcium levels to exceed the normal limits. The high production of parathyroid hormone (PTH) and intracellular calcium levels will trigger the release of angiotensin II, which is a factor contributing to the contraction of blood vessels. In addition to triggering the release of angiotensin II, this situation will lead to increased secretion of calci-tonin. As a result, the re-absorption of calcium by the kidneys and digestive system will decrease which will result in increased excretion of calcium through the urine so that no mineralization of new bone and cartilage matrix is causes bones to become brittle. ${ }^{11,12}$

Based on the research that has been done the average value of the percentage trabecular area (radiopaque) is much lower than the average value of the percentage marrow area (radiolucent). This suggests a widening cavity that causes the trabecular bone mass in each volume will be reduced, resulting in a change in the trabecular density. The results are consistent with research conducted by Azhari et al. ${ }^{2}$ which states the trabecular pattern comprehensive overview of radiolucent as marrow larger than the radiopaque as trabecular in patients with osteopenia and osteoporosis. This study also contradict the theory that healthy trabecular compact with small radiolucent cavities therebetween. ${ }^{2,14}$

Based on the principles of radiographic interpretation, radiopaque picture is the picture that the arrangement of the bones contain the most calcium. Based on the results in table 4 shows the average trabecular area (radiopaque) in hypertensive patients is lower than marrow area (radiolucent) in patients with hypertension. This indicates that in patients with hypertension calcium on bone formation is lower. Several studies have concluded that the trabecular bone density associated with bone density in other body parts. The imbalance of bone resorption and bone deposits systemically very likely to manifest more quickly in the condyle than in other parts, because the trabecular have a high metabolism so that it can occur up to 5-8 times compared with cortical parts. Changes that occur on the surface of trabecular that is trabecular thinning, trabecular perforation plate types, reduced branching trabecular and marrow area expansion. ${ }^{13,14}$

The results of this study showed the average value of the percentage trabecular bone left and right condyle patients with hypertension have a low value, that is $29.720 \%$ and $30.138 \%$ Which measures the average normal trabecular bone in the region of condyle with panoramic radiograph, which amounted to $44.050 \%$. This indicates that in patients with hypertension tend to have reduced bone density changes. ${ }^{15}$

Panoramic radiographs can be used to determine the quality of mandibular bone that can be associated with bone mass density (BMD) in the vertebrae. ${ }^{16}$ Panoramic radiography can perform all the pictures of mandibular in a film so that it can be used to see the changes in trabecular bone density. ${ }^{17-19}$ The results are consistent with research conducted by Watanabe et al. ${ }^{3}$ states that panoramic radiographs can assess bone density in microstructure and can be evaluated through the trabecular bone. The research was supported by White et al. ${ }^{18}$ which states that changes in trabecular structure was detected on dental radiographs. ${ }^{3,18}$

Research Shimamoto. ${ }^{1}$ based on trabecular also proven in animal experiments based on analysis of trabecular patterns and can detect changes in the bone quality by $94 \%$, while based on cortical only by $64 \%$. Software ImageJ is a software that used in the research to determine the percentage trabecular bone density of each data panoramic radiographs patients with hypertension. The change in the density of the radiograph can be helpful in determining the treatment plan and prevention of complications that would be detrimental to the patient. ${ }^{1,3}$

\section{Conclusion}

The conclusions of this study is the condyle trabecular bone density of hypertension patients evaluated from panoramic radiographs using software ImageJ tends to decrease. The results of this study are expected to be the basis of further research by developing research methods to determine the relationship of hypertension and bone density. Moreover, it can add a variable to determine its relationship with bone density.

\section{Acknowledgment}

None. 


\section{Conflict of Interest}

The authors report no conflict of interest.

\section{References}

1. Shimamoto. Radiological morphometry analysis for the trabecular bone structure of mandibular condyle after ovariectomy in mature cynomolgus monkeys. Oral Sci Int 2007;4: 86-96.

2. Azhari, Suprijanto, Diputra Y, et al. Analysis of panoramic radiographic images of the mandibular bone for early detection of osteoporosis using the gray level cooccurence matrix (GLCM) method. Indonesian J App Sci 2014;46: 203-208. (In Indonesia)

3. Watanabe PCA, Farman A, Watanabe MGDC, et al. Radiographic signals detection of systemic disease. Int J Morphol 2008;26: 915-926.

4. Manurung N. Cardiovascular system nursing care application. Jakarta Timur: CV. Trans Info Media; 2016. p. 102. (In Indonesian)

5. Depkes RI. Basic Health Research. Kemenkes RI: Jakarta. 2013. (In Indonesia)

6. Primadita. The relationship between intake of sodium, potassium, magnesium, and calcium with hypertension at the Puskesmas Mergangsan Yogyakarta. J Kedokteran \& Kesehatan 2009;7: 52-60. (In Indonesian)

7. Permatasari, Tria AE. The relationship between calcium intake and other risk factors with the incidence of osteoporosis in the early adult group in the ciputatSouth Tangerang. J Kedokteran \& Kesehatan 2011;7: 35-44. (In Indonesian)

8. Fitrianto H, Azmi S, Kadri H. The use of antihypertensive drugs in patients with essential hypertension in hypertensive renal polyclinic RSUP DR. M. Djamil in 2011. J Kesehatan Andalas 2014;3: 45-48. (In Indoensian)

9. Ullah MI, Uwaifo GI, Nicholas WC, et al. "Does vitamin D deficiency cause hypertension? Current evidence from clinical studies and potential mechanisms". Int J Endocrinol 2010.

10. Bailer W. Writing imagej plugins a tutorial. Upper Austria University App Sci 2006;1-57.
11. Kurusu A, Horiuchi M, Soma K. Relationship between occlusal force and mandibular condyle morphology (evaluated by limited cone-beam computed tomography). Angle Orthod 2009;79: 1063-1069.

12. Saufika A, Retnaningsih, Alfiasari. Student lifestyle and eating habits. J Ilmu Keluarga \& Konsumen 2012;5: 157-165. (In Indonesian)

13. Lanham-New SA. The balance of bone health: tipping the scales in favor of potassium-rich, bicarbonate-rich foods. J Nurit 2008: 172-177.

14. Eijden TMGJ, LJ Van Ruijven, Giesen EBW. Bone Tissue Stiffness in the mandibular condyle is dependent on the direcion and density of the cancellouse structure. Calcif Tissue Int 2004;75: 502-550.

15. Jie-Yang, Pham SM, Crabbe DL. Effects of estrogen deficiency on rat mandibular and tibial microarchitecture. J Med Oral Patol Oral Cir Bucal 2012;17: 624-632.

16. Azhari, Suprijanto, Prafiadi H, et al. Analysis of the ability of panoramic radiographic images in detecting bone trabecular density using micro-CT as the standard. Indonesian J App Sci 2014;4: 1-5. (In Indonesian)

17. Lestari S, Suparta GB, Kertia N. The correlation between texture parameter of mandible trabecullar bone with the bone mass density value. Proc. Of IEEE, The 7th ICBEMA 2012;4: 44-52.

18. White SC, Atchinson KA, Gornbein JA, et al. Change in mandibular trabecular pattern and hip fracture rate in elderly women. Dentomaxillofac Radiol 2005;34: 168-174.

19. Nurwahida, Hardianto A, Rizki KA. Multiple sialolithiasis of submandibular gland: a case report. J Dentomaxillofac Sci 2017;2: 129-132.

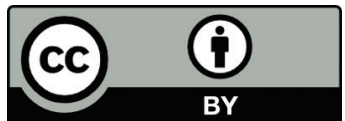

This work is licensed under a Creative Commons Attribution 